\title{
Reproducibility of Three-Dimensional Retinal Organoids as In Vitro Models of Human Retinal Development and Organization
}

\author{
Elyse Feder ${ }^{1}$, Jade Harkin ${ }^{1}$, Jason Meyer ${ }^{1,2,3}$ \\ ${ }^{1}$ Indiana University School of Medicine, ${ }^{2}$ Department of Medical and Molecular Genetics, ${ }^{3}$ Stark \\ Neurosciences Research Institute
}

Humans have limited ability to repair damaged central nervous system neurons, such as retinal ganglion cells, making it critical to advance towards ways to reconstruct a patient's retina if one suffers from trauma or a neurodegenerative disease of the eye, such as glaucoma and other blinding disorders. The use of human pluripotent stem cells (hPSCs) provides powerful approaches to the development of novel therapeutic strategies to blinding diseases due to their ability to self-renew and form any cell type in the human body through targeted differentiation. Recently, hPSCs have been shown to self-form retinal organoids, which are three-dimensional aggregates of cells that mimic the spatial and temporal organization of the human retina in vitro. Therefore, retinal organoids provide an in vitro model of the human retina for drug screening, disease modeling, and cell replacement therapies.

However, before these approaches can be fully realized, a critical need exists to refine these differentiation methods as current protocols lead to heterogeneity of organoids produced, as well as inconsistency in their shape and size, leading to significant variability within experiments. Thus, the goal of this research is to optimize current retinal organoid differentiation protocols to generate highly reproducible retinal organoids that are consistent in size, shape and cellular composition across various stem cell lines that can be used for disease modeling and highthroughput applications. The size and shape of retinal organoids was experimentally controlled for through the use of low-adhesion 96 well U-bottom plates with centrifugation to induce quick reaggregation of individual undifferentiated hPSCs. These aggregates were compared to those generated through traditional methods in which whole colonies were mechanically lifted.

Resulting retinal organoids at early and later stages showed greater reproducibility in size and shape across different stem cell lines, demonstrating greater suitability for direct comparisons in disease modeling and drug screening experiments. 